\title{
Molecular Evidence of Drug Resistance in Asymptomatic Malaria Infections, Myanmar, 2015
}

\author{
Myat Htut Nyunt, Thinzar Shein, Ni Ni Zaw, \\ Soe Soe Han, Fauzi Muh, Seong-Kyun Lee, \\ Jin-Hee Han, Kyaw Zin Thant, \\ Eun-Taek Han, ${ }^{1}$ Myat Phone Kyaw ${ }^{1}$
}

Artemisinin resistance containment in Myanmar was initiated in 2011 after artemisinin-resistant Plasmodium falciparum malaria was reported. Molecular evidence suggests that asymptomatic malaria infections harboring drug resistance genes are present among residents of the Myanmar artemisinin resistance containment zone. This evidence supports efforts to eliminate these hidden infections.

$\mathrm{T}$ he global burden of malaria has been decreasing in recent years as a result of high levels of control of the spread of infection, and the ultimate goal of malaria elimination by 2030 in all Greater Mekong Subregion countries in Southeast Asia seems attainable (1). However, artemisinin-resistant Plasmodium falciparum malaria has been reported in Cambodia, Thailand, Myanmar, Laos, and Vietnam (2). Chloroquine-resistant $P$. vivax malaria has also been confirmed in 10 countries, including Myanmar (3), and mutations in the mefloquine-resistance molecular marker (pvmdr1 mutation) and sulfadoxine/pyrimethamine-resistance markers ( $p v d h p s, p v d h f r$ mutations) have been reported in Myanmar (4).

A containment program for artemisinin-resistant malaria was initiated in 2011 according to the Global Plan for Artemisinin Resistance Containment. Areas where artemisinin resistance was documented were ranked as Tier I under the protocol, whereas areas where resistance was suspected were ranked as Tier II. After Myanmar artemisinin resistance containment (MARC) was initiated, malaria morbidity and mortality rates decreased dramatically, especially in MARC Tier I areas (5). However, there are no reports on the prevalence of asymptomatic infections, which may represent a reservoir of local malaria transmission. In this study, we aimed to determine the prevalence of asymptomatic malaria infection and to analyze drug-resistance markers in asymptomatic $P$. falciparum and $P$. vivax infections.

Author affiliations: Department of Medical Research, Yangon, Myanmar (M.H. Nyunt, T. Shein, N.N. Zaw, S.S. Han, K.Z. Thant, M.P. Kyaw); Kangwon National University, Chuncheon, South Korea (M.H. Nyunt, S.-K. Lee, J.-H. Han, F. Muh, E.-T. Han)

\section{The Study}

As of 2014, the Tier I area of artemisinin resistance in Myanmar was composed of 52 townships; the remaining regions were designated as Tier II. In January 2015, we conducted a cross-sectional study of one of the Tier I areas of the MARC, Shwegyin Township $\left(22^{\circ} 20^{\prime} 0^{\prime \prime} \mathrm{N}\right.$, $95^{\circ} 56^{\prime} 0^{\prime \prime} \mathrm{E}$ ) (Figure; online Technical Appendix, https:// wwwnc.cdc.gov/EID/article/23/3/16-1363-Techapp1.pdf).

Rapid diagnostic tests (RDTs) (HRP2 and P. vivaxspecific pLDH-based RDT, SDFK80; Standard Diagnostics, Gyeonggi-do, South Korea), microscopy, and PCR were used to screen for asymptomatic malaria infection (online Technical Appendix). We examined 1,182 local residents, with a male:female ratio of $4: 5$ and a median age of 30 years (interquartile range 18-45 years). Among these residents, $549(46.4 \%)$ had a history of malaria infection within the past 5 years. No clinical cases of malaria infection were detected during the survey period. Although we found no RDT-positive cases of malaria infection, we detected $2 P$. vivax infections by microscopy, with parasite densities of 580 and 1,200 parasites $/ \mu \mathrm{L}$.

When we performed molecular detection for the 4 common malaria species (online Technical Appendix), the overall rate of asymptomatic malaria infection was $2.4 \%$ $(28 / 1,180)$ and included $4 P$. falciparum, $22 P$. vivax, and 2 $P$. malariae infections. Although the overall prevalence of asymptomatic infection in these areas was not high, it was similar to that observed in the Thailand-Myanmar border area during 2013-2014 (6).

In this study, RDT and microscopy missed almost all the asymptomatic infections detected by PCR, indicating that only the molecular method is suitable for the detection of asymptomatic infections. Moreover, the asymptomatic cases were broadly distributed geographically throughout the study area. Most of the infections were in male patients $(19 / 28,67.8 \%)$ and in the working-age group. Neither sex nor occupation was identified as an associated factor for asymptomatic infection (online Technical Appendix Table 2).

The established artemisinin-resistance marker K13 (kelch 13 gene) and the associated markers pfarps $10(P$. falciparum apicoplast ribosomal protein S10), pffd ( $P$. falciparum ferredoxin), and $p f m d r 2$ ( $P$. falciparum multidrugresistance protein 2 ) were analyzed in all asymptomatic

${ }^{1}$ These authors contributed equally to this article. 


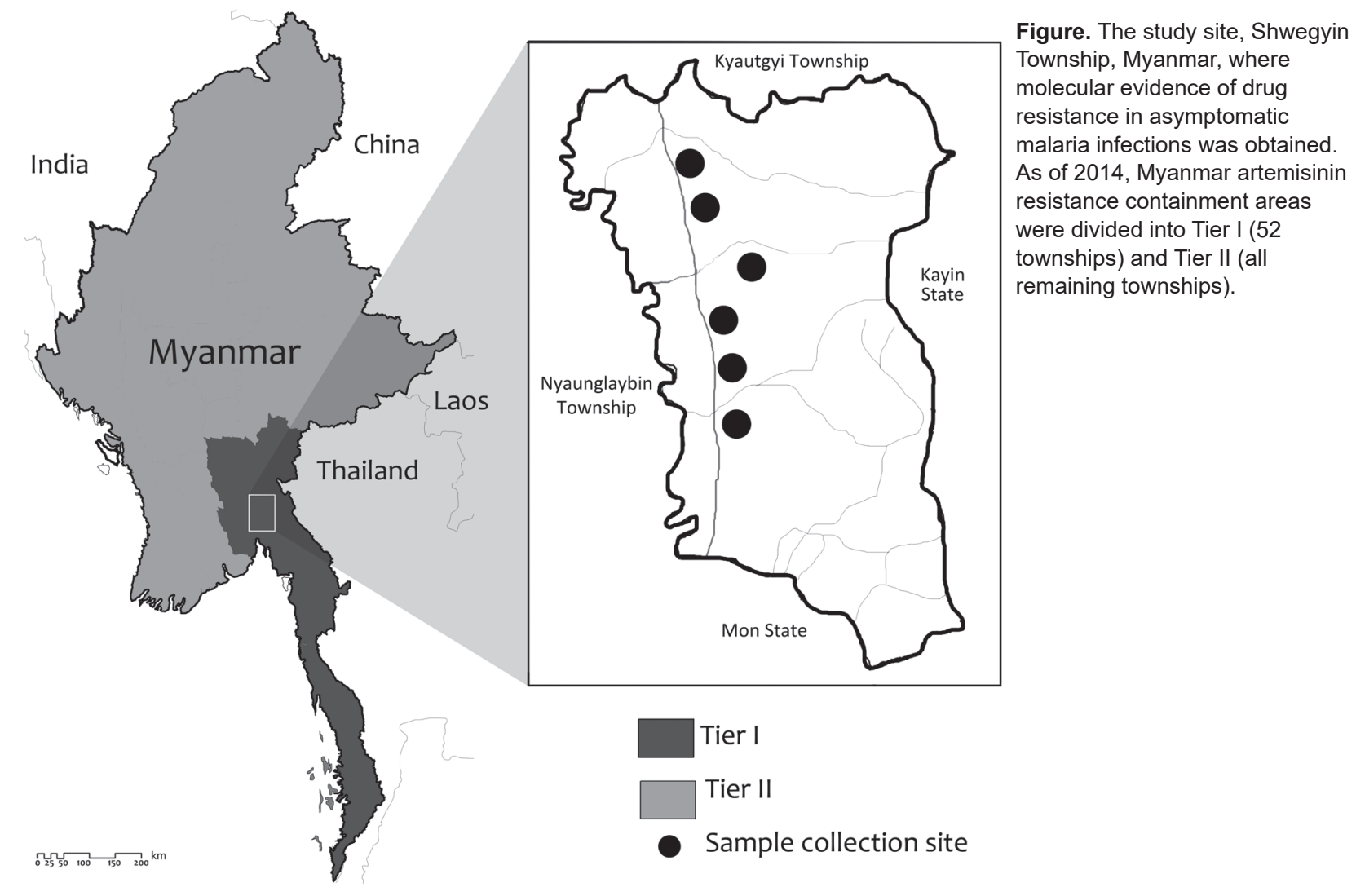

P. falciparum cases. Nonsynonymous mutations in the propeller region of K13 were found to be associated with artemisinin resistance and associated delayed clearance of the parasite beyond 72 hours after treatment with artemisinin-based combination therapy (7). A previous study in the same region of patients with uncomplicated $P$. falciparum malaria indicated that $25.3 \%$ carried mutant K13 alleles (8). Markers that showed the underlying genetic background predisposing to the K13 mutant were also reported, including pfarps 10, pffd, pfmdr2, and pfcrt. Specific single nucleotide polymorphisms of these genes, such as V127M of pfarps10, D193Y of $p f f d$, and T484I of $p f m d r 2$, were found at a similar prevalence as K13 mutations (9).

Among the 4 asymptomatic $P$. falciparum infections, 2 isolates showed $\mathrm{K} 13$ mutations $(\mathrm{C} 580 \mathrm{Y}$ in 1 isolate and $\mathrm{P} 574 \mathrm{~L}$ in the other). C580Y is a well-known validated mutation, and $\mathrm{P} 574 \mathrm{~L}$ is a candidate marker for artemisinin resistance. Both mutations were reported only in locations in Southeast Asia where artemisinin resistance has been identified (2). Moreover, we observed the pfarps 10 mutation (V127M) in 2 of the cases, the $p f f d$ mutation (D195Y) in 3 cases, and the pfmdr2 (T484I) mutation in all 4 isolates (Table; online Technical Appendix Table 3). This molecular evidence suggests the presence of artemisinin resistance in asymptomatic isolates and calls for action toward eliminating this parasite reservoir.
Similarly, we analyzed all available drug-resistance molecular markers in P. vivax (10), such as pvcrt ( $P$. vivax chloroquine-resistance transporter), pvdhps ( $P$. vivax dihydropteroate synthase), pvdhfr ( $P$. vivax dihydrofolate reductase), and pvmdrl ( $P$. vivax multidrug-resistance protein 1), in all $P$. vivax infections. We conducted analysis by using nested PCR, followed by gene sequencing (online Technical Appendix).

Among the 22 asymptomatic $P$. vivax infections, we were unable to amplify pvcrt-o, pvdhfr, and pvmdrl in 1 isolate and pvdhps in 2 isolates. A high mutation rate was observed in known drug-resistance markers such as pvcrt-o K10 AAG insert $(66.6 \%, 14 / 21)$, pvdhps $(100.0 \%, 20 / 20)$, pvdhfr $(100.0 \%, 21 / 21)$, and pvmdr1 $(100.0 \%, 21 / 21)$ (Table). Asymptomatic isolates in this study showed a higher mutation rate of the pvcrt-o AAG insert than those studied in neighboring countries such as Thailand (11), India (12), and China (10). In the pvmdr1 gene, both Y976F and F1076L mutations were observed in $23.8 \%$ of cases and $\mathrm{F} 1076 \mathrm{~L}$ in $19.0 \%$ of cases; these rates were higher than those for China (10) and India (12) but lower than those for Thailand $(13,14)$. Although antifolates are not the recommended antimalarial drugs for treatment of $P$. vivax, $p v d h f r$ and $p v d h p s$ mutation rates were noticeable. This finding indicates that drug pressure in $P$. vivax malaria contributing to drug resistance also 
Table. Plasmodium falciparum and $P$. vivax drug-resistance molecular markers in asymptomatic infections, Myanmar, 2015

\begin{tabular}{|c|c|c|}
\hline Target & Description* $^{*}$ & No. isolates/total (\%) \\
\hline \multirow[t]{3}{*}{ kelch $13(\mathrm{~K} 13)$} & Wild & $2 / 4(50.0)$ \\
\hline & C580Y & $1 / 4(25.0)$ \\
\hline & P574L & $1 / 4(25.0)$ \\
\hline \multirow{2}{*}{$\begin{array}{l}\text { P. falciparum apicoplast ribosomal protein S10 } \\
\text { (pfarps10) }\end{array}$} & Wild & $2 / 4(50.0)$ \\
\hline & V127M & $2 / 4(50.0)$ \\
\hline \multirow[t]{2}{*}{ P. falciparum ferredoxin $(p f f d)$} & Wild & $1 / 4(25.0)$ \\
\hline & D193Y & $3 / 4(75.0)$ \\
\hline \multirow[t]{2}{*}{ P. falciparum multidrug-resistance protein 2 (pfmdr2) } & Wild & $0 / 4(0.0)$ \\
\hline & T484I & $4 / 4(100.0)$ \\
\hline \multirow[t]{2}{*}{ P. vivax chloroquine-resistance transporter ( $p v c r t-o)$} & Wild & $7 / 21(33.3)$ \\
\hline & Mutant (AAG insert) & $14 / 21(66.7)$ \\
\hline \multirow[t]{4}{*}{ P. vivax multidrug-resistance protein 1 (pvmdr1) } & Wild $(T, Y, F)(958,976,1076)$ & $0 / 21(0.0)$ \\
\hline & Double mutant $(\mathbf{M}, \mathbf{Y}, \mathbf{L})$ & $4 / 21(19.0)$ \\
\hline & Single mutant $(M, Y, F)$ & $12 / 21(57.1)$ \\
\hline & Triple mutant $(\mathbf{M}, \mathbf{F}, \mathbf{L})$ & $5 / 21(23.8)$ \\
\hline \multirow[t]{5}{*}{ P. vivax dihydropteroate synthase (pvdhps) } & Wild $(\mathrm{S}, \mathrm{A}, \mathrm{K}, \mathrm{A})(382,383,512,553)$ & $0 / 20(0.0)$ \\
\hline & Single mutant $(\mathrm{S}, \mathbf{G}, \mathrm{K}, \mathrm{A})$ & $4 / 20(20.0)$ \\
\hline & Double mutant (S, G, K, G) & $9 / 20(45.0)$ \\
\hline & Triple mutant (A, G K K G) & $5 / 20(25.0)$ \\
\hline & Quadruple mutant $(\mathbf{A}, \mathbf{G}, \mathbf{M}, \mathbf{G})$ & $2 / 20(10.0)$ \\
\hline \multirow[t]{4}{*}{ P. vivax dihydrofolate reductase ( $p v d h f r)$} & Wild $(F, S, T, S)(57,58,61,117)$ & $0 / 21(0.0)$ \\
\hline & Single mutant $(\mathrm{L}, \mathrm{S}, \mathrm{T}, \mathrm{S})$ & $1 / 21(4.8)$ \\
\hline & Double mutant $(\mathrm{F}, \mathbf{R}, \mathrm{T}, \mathbf{N})$ & $2 / 21(9.5)$ \\
\hline & Quadruple mutant (L/I, R, M, T) & $18 / 21(85.7)$ \\
\hline
\end{tabular}

needs to be considered in addition to emphasizing the artemisinin-resistant $P$. falciparum malaria.

One limitation of this study is the exclusive focus on the local residents in the MARC area, where all available control and prevention measures had already been implemented. Unlike the mobile and migrant population, local residents have not been a top priority for the artemisinin resistance containment program, leading to a niche of hidden infection. Moreover, blood pooling before DNA extraction was used in this study for molecular detection of malaria infection. Although this method is not ultrasensitive, it has a higher sensitivity than RDT and microscopy. The hidden asymptomatic infections and associated molecular markers for drug resistance among the asymptomatic cases detected in this study represent a threat to containment and elimination efforts with regard to drug-resistant parasites.

\section{Conclusions}

All countries in the Greater Mekong Subregion have set an ultimate goal of eliminating malaria by 2030 . One of the main challenges to achieving this goal is hidden asymptomatic infection, which maintains a reservoir for local transmission of malaria (15). Critically, these asymptomatic infections may carry drug-resistance genes, including genes for artemisinin resistance. Our results indicated that drug-resistant malaria parasites may be spreading, even in the containment areas or (pre-)elimination areas; this issue should, therefore, be addressed at a policy level. Detection and elimination of asymptomatic infections are of vital importance. Our evidence highlights the need for a strategy for eliminating drug-resistant malaria in asymptomatic infections in the containment areas.

\section{Acknowledgments}

We thank all participants in this study and local health authority personnel and staff from the Parasitology Research Division and Advanced Molecular Research Center, Department of Medical Research and Kangwon Malaria Research Lab, Kangwon National University, South Korea.

This study was supported by the Korea Association of Health Promotion (2016_02), the National Research Foundation of Korea (NRF) grant funded by the South Korea government (MSIP) (NRF-2014R1A2A1A11052079), and by the Basic Science Research Program through the NRF funded by the Ministry of Science, ICT and Future Planning (2015R1A4A1038666).

Dr. Nyunt is a research scientist in the Department of Medical Research, Republic of the Union of Myanmar, and is currently studying in Kangwon National University, Chuncheon, South Korea. His research interests include drug-resistant malaria and neglected tropical diseases.

\section{References}

1. World Health Organization. Strategy for Malaria Elimination in the Greater Mekong Subregion (2015-2030). WHO Regional Office for the Western Pacific; 2015 [cited 2016 Aug 16]. http://www.wpro.who.int/mvp/documents/strat_mal_elim_gms/en/

2. World Health Organization. Status report: Artemisinin and artemisinin-based combination therapy resistance. Geneva: The Organization; 2016 [cited 2016 Aug 16]. http://apps.who.int/iris/ handle $/ 10665 / 208820$ 
3. World Health Organization. World Malaria Report 2015. Geneva: The Organization; 2015 [cited 2016 Aug 16]. http://www.who.int/ malaria/publications/world-malaria-report-2015/report/en/

4. World Health Organization. Global report on antimalarial drug efficacy and drug resistance: 2000-2010. Geneva: The Organization; 2010 [cited 2016 Aug 16]. http://www.who.int/malaria/publications/ atoz/9789241500470/en/

5. Township Health Department. Township Health Profile 2012: Shwegyin Township. Department of Health, Myanmar; 2013.

6. Baum E, Sattabongkot J, Sirichaisinthop J, Kiattibutr K, Jain A, Taghavian O, et al. Common asymptomatic and submicroscopic malaria infections in western Thailand revealed in longitudinal molecular and serological studies: a challenge to malaria elimination. Malar J. 2016;15:333. http://dx.doi.org/10.1186/s12936-016-1393-4

7. Ariey F, Witkowski B, Amaratunga C, Beghain J, Langlois AC, Khim N, et al. A molecular marker of artemisinin-resistant Plasmodium falciparum malaria. Nature. 2014;505:50-5. http://dx.doi.org/10.1038/nature12876

8. Ashley EA, Dhorda M, Fairhurst RM, Amaratunga C, Lim P, Suon S, et al.; Tracking Resistance to Artemisinin Collaboration (TRAC). Spread of artemisinin resistance in Plasmodium falciparum malaria. N Engl J Med. 2014;371:411-23. http://dx.doi.org/10.1056/NEJMoa1314981

9. Miotto O, Amato R, Ashley EA, MacInnis B, Almagro-Garcia J, Amaratunga $\mathrm{C}$, et al. Genetic architecture of artemisininresistant Plasmodium falciparum. Nat Genet. 2015;47:226-34. http://dx.doi.org/10.1038/ng.3189

10. Lu F, Wang B, Cao J, Sattabongkot J, Zhou H, Zhu G, et al Prevalence of drug resistance-associated gene mutations in Plasmodium vivax in Central China. Korean J Parasitol. 2012;50:379-84. http://dx.doi.org/10.3347/kjp.2012.50.4.379
11. Lu F, Lim CS, Nam D-H, Kim K, Lin K, Kim T-S, et al Genetic polymorphism in pvmdr1 and pvcrt-o genes in relation to in vitro drug susceptibility of Plasmodium vivax isolates from malaria-endemic countries. Acta Trop. 2011;117:69-75. http://dx.doi.org/10.1016/j.actatropica.2010.08.011

12. Ganguly S, Saha P, Guha SK, Das S, Bera DK, Biswas A, et al. In vivo therapeutic efficacy of chloroquine alone or in combination with primaquine against vivax malaria in Kolkata, West Bengal, India, and polymorphism in pvmdrl and pvcrt-o genes. Antimicrob Agents Chemother. 2013;57:1246-51. http://dx.doi.org/10.1128/ AAC.02050-12

13. Suwanarusk R, Chavchich M, Russell B, Jaidee A, Chalfein F, Barends M, et al. Amplification of pvmdrl associated with multidrug-resistant Plasmodium vivax. J Infect Dis. 2008;198:1558-64. http://dx.doi.org/10.1086/592451

14. Rungsihirunrat K, Muhamad P, Chaijaroenkul W, Kuesap J, Na-Bangchang K. Plasmodium vivax drug resistance genes; Pvmdrl and Pvcrt-o polymorphisms in relation to chloroquine sensitivity from a malaria endemic area of Thailand. Korean J Parasitol. 2015;53:43-9. http://dx.doi.org/10.3347/ kjp.2015.53.1.43

15. Okell LC, Ghani AC, Lyons E, Drakeley CJ. Submicroscopic infection in Plasmodium falciparum-endemic populations: a systematic review and meta-analysis. J Infect Dis. 2009;200:1509-17. http://dx.doi.org/10.1086/644781

Address for correspondence: Eun-Taek Han, Department of Medical Environmental Biology and Tropical Medicine, Kangwon National University, Chuncehon-si, Gangwon-do, 200-701, South Korea; email: ethan@kangwon.ac.kr; etaekhan@gmail.com

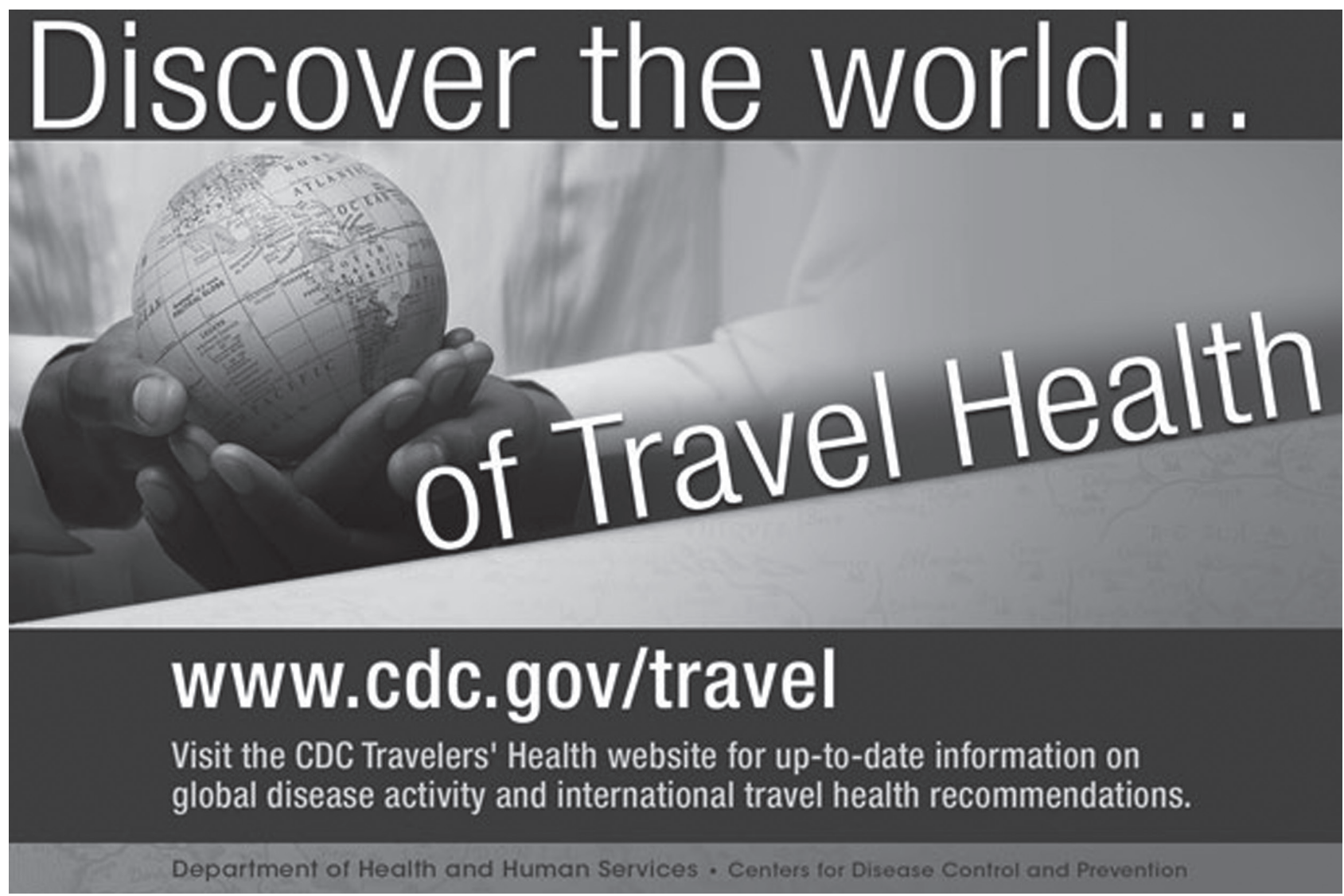

